\title{
Comparing the response modulation hypothesis and the integrated emotions system theory: The role of top-down attention in psychopathy
}

\author{
Jaap Munneke ${ }^{\mathrm{a}, \mathrm{b}, \mathrm{c}, *}$, Sylco S. Hoppenbrouwers ${ }^{\mathrm{d}}$, Bethany Little ${ }^{\mathrm{c}}$, Karen Kooiman ${ }^{\mathrm{c}}$, \\ Erik van der Burg ${ }^{c, e}$, Jan Theeuwes ${ }^{c}$ \\ a Aysel Sabuncu Brain Research Center, Bilkent University, Ankara, Turkey \\ b Department of Psychology, Bilkent University, Ankara, Turkey \\ ${ }^{c}$ Department of Experimental and Applied Psychology, Vrije Universiteit Amsterdam, Netherlands \\ d Department of Clinical Psychology, Erasmus University Rotterdam, Netherlands \\ e School of Psychology, University of Sydney, Australia
}

\section{A R T I C L E I N F O}

\section{Keywords:}

Psychopathy

Response modulation

Attention

Emotional capture

Fear

\begin{abstract}
A B S T R A C T
Objective: Two major etiological theories on psychopathy propose different mechanisms as to how emotional facial expressions are processed by individuals with elevated psychopathic traits. The Response Modulation Hypothesis (RMH) proposes that psychopathic individuals show emotional deficits as a consequence of attentional deployment, suggesting that emotional deficits are situation-specific. The Integrated Emotions System theory (IES) suggests that psychopathic individuals have a fundamental amygdala dysfunction which precludes adequate responsiveness to the distress of others.

Methods: Participants performed a visual search task in which they had to find a male target face among two female distractor faces. Top-down attentional set was manipulated by having participants either respond to the face's orientation, or its emotional expression.

Results: When emotion was task-relevant, the low-scoring psychopathy group showed attentional capture by happy and fearful distractor faces, whereas the elevated group showed capture by fearful, but not happy distractor faces.

Conclusion: This study provides evidence for the RMH such that top-down attention influences the way emotional faces attract attention in individuals with elevated psychopathic traits. However, the different response patterns for happy and fearful faces suggest that top-down attention may not determine the processing of all types of emotional facial expressions in psychopathy.
\end{abstract}

\section{Introduction}

Psychopathy is a well-known personality disorder, characterized by aberrant emotionality and antisocial behavior. Particularly well-known are the unemotional and callous personality traits of psychopathic individuals, often accompanied by antisocial behavior (e.g., criminal behavior and poor behavioral control; Blair \& Mitchell, 2009; Hare, 1991; Hoppenbrouwers, Bulten, \& Brazil, 2016).

Recent work has shown an increased interest in how psychopathic individuals perceive and interpret emotional facial expressions. Previous studies have revealed a diverse pattern of results concerning the ability of psychopathic individuals to recognize, process and act upon varying emotional facial expressions such as happy, fearful and angry (Fairchild, Van Goozen, Calder, Stollery, \& Goodyer, 2009; Fairchild, Stobbe, van Goozen, Calder, \& Goodyer, 2010; but see
Glass \& Newman, 2006). Diverse results notwithstanding, converging evidence has suggested that the manner in which emotional expressions are processed by psychopathic individuals is qualitatively different from those processes in healthy individuals (Dawel, O'Kearney, McKone, \& Palermo, 2012; Marsh \& Blair, 2008). However, the underlying mechanisms leading to differences in processing emotional expressions in psychopathic individuals are poorly understood. While deficits in affective functioning have been hypothesized to reflect the core of psychopathy, aberrations in cognitive factors such as attention have also been put forth as important etiological factors (BaskinSommers, Curtin, \& Newman, 2011; Newman \& Baskin-Sommers, 2012). These opposing views are reflected in two theories that aim to describe the mechanisms underlying psychopathy.

First, the Response Modulation Hypothesis (RMH) proposes a malfunction in information-processing abilities as an important contributor

\footnotetext{
* Corresponding author at: Aysel Sabuncu Brain Research Center, Bilkent University, 06800, Bilkent, Ankara, Turkey.

E-mail address: Jacob.Munneke@bilkent.edu.tr (J. Munneke).
} 
to psychopathic behavior. Specifically, psychopathic behavioral traits are suggested to originate from deficits in the ability to rapidly switch from goal-directed behavior to attending task-irrelevant information when processing this irrelevant information could lead to beneficial behavior or improved social interaction (Gorenstein \& Newman, 1980; Newman \& Wallace, 1993; Patterson \& Newman, 1993). According to Newman and Wallace (1993) psychopathic individuals are deficient in detecting and redirecting top-down attention towards important or informative, yet task-irrelevant stimuli, resulting in the non-adaptive perseverance of behavioral patterns solely aimed at ongoing goals. Indeed, evidence for the RMH is observed in a host of studies emphasizing the presence of attentional abnormalities in psychopathy (e.g. BaskinSommers et al., 2011; Hoppenbrouwers, Van der Stigchel, Sergiou, \& Theeuwes, 2016; Hoppenbrouwers, van der Stigchel, Slotboom, Dalmeijer, \& Theeuwes, 2015; Newman, Curtin, Bertsch, \& Baskin-Sommers, 2010). In short, the RMH states that psychopathic individuals have a rigid, inflexible mechanism of top-down attentional control, resulting in diminished attentional resources being allocated to information not fitting the psychopathic individual's topdown set.

A second theory on the etiological factors of psychopathy is the Integrated Emotions System theory (IES; Blair, 2005) which focuses on deficits in the processing of emotional and affective stimuli. At the focus of the IES lies a dysfunction in the amygdala (Blair et al., 2004), which may prevent the allocation of attention towards emotional expressions. Amygdala deficits are hypothesized to cause an impairment in the formation of aversive stimulus-reinforcement associations in psychopathic individuals, with aversive stimuli extending to emotional expressions such as fear and anger (Blair, 2005). As a consequence, emotions that signal distress in others are not processed effectively by psychopathic individuals, resulting in diminished social behavior.

The RMH and IES lead to a number of opposing hypotheses concerning how emotional facial expressions may influence attentional selection. First, the RMH's focus on attentional processes as the source of psychopathic behavior predicts that aberrant behavior by psychopathic individuals is situation specific rather than pan-situational: Only when threat-signaling stimuli or emotional expressions do not match the top-down goals of the psychopathic individual will these stimuli go unnoticed and unattended. In short, it is the relevance of a stimulus that matters. On the contrary, the IES makes no claims about the relevance of stimuli and predicts that psychopathic individuals are generally unresponsive to aversive emotional stimuli due to disrupted amygdala functioning. A consequence of the lack of emotional responsivity to aversive stimuli is that attention may not be automatically drawn to such emotional stimuli and as such this emotional information is not, or to a lesser extent processed. Second, according to the RMH, the deficiency in processing non-relevant information does not only pertain to threat-signaling or emotional stimuli, but to any type of stimulus that does not match the psychopathic individual's goals and as such remains unattended. Alternatively, the IES does not make such a broad claim by stating that attentional selection in psychopathic individuals is predominantly determined by the absence of a stimulus-reinforcement relation between an aversive stimulus and a behavioral response towards that stimulus. The IES therefore makes specific predictions that tailor to aversive stimuli such as facial expressions signaling distress, but does not make any claims regarding the relevance of such information. Regarding attention, the IES predicts differences between aversive emotional expressions such as fearful faces, as compared to neutral or positive expressions, regardless of whether processing these emotions is part of the psychopathic individual's top-down set.

To investigate how attention to emotional expressions is altered in psychopathy and to distinguish between the RMH and the IES, we employed a paradigm used by Hodsoll, Lavie, and Viding (2014). Hodsoll and colleagues investigated how emotional facial expressions affected the allocation of attention in children with high versus low callous-unemotional traits (CU-traits; a precursor for psychopathy:
Viding \& McCrory, 2012) and healthy controls (for more information on the influence of emotion on attentional processes see Byrne \& Eysenck, 1995; Yiend, 2010). In their paradigm, participants searched for a male target face among two female distractor faces and indicated whether the male face was tilted to the left or the right. On a subset of trials, one of the faces contained an emotional expression, either presented on the male target face or on one of the two female distractor faces. This manipulation allowed for testing the difference in attentional allocation to emotional facial expressions in a high CU group, a low CU group and healthy controls using both emotional distractor faces and emotional target faces. Results showed that children with increased callous-unemotional traits showed reduced attentional capture by irrelevant emotional faces; a finding in support of the IES.

In the current study, we added a critical condition in which participants had to judge the emotion of a male target face, rather than its orientation. Using both positive and negative emotional expressions under different task demands was done for multiple reasons: First, the IES predicts that individuals with elevated psychopathic traits will show an abnormality in the detection of a fearful facial expression, irrespective of the task set (i.e., whether the participant is responding to the emotion or the orientation). As such, it is expected that individuals with elevated psychopathic traits are slower than controls when the target face displays a fearful facial expression. However, when a distractor displays a fearful facial expression, the IES predicts that individuals with elevated psychopathic traits show better performance than controls because they are not influenced by the fearful expression on the distractor. Second, contrary to the IES, the RMH predicts that emotional differences between individuals with low versus elevated psychopathic traits arise as a function of task-relevance, independent of the emotion's valence. Therefore, during the emotion task, the RMH predicts no difference between healthy controls and psychopathic individuals as emotion is always part of an individual's top-down set. On the contrary, during the orientation task, emotion is task irrelevant. Emotional expressions may still automatically capture attention in healthy controls, but not in psychopathic individuals as their top-down set is focused on orientation, rather than the emotion of the presented stimuli. By manipulating top-down attentional set and using different emotional facial expressions we directly compare the RMH and the IES with the aim of reconciling earlier contrasting findings.

\section{Methods}

\subsection{Participants}

We tested a mixed community sample $(N=100)$ consisting of 80 undergraduate students and 20 non-students recruited from the community (36 males, mean age $=24.4, \mathrm{SD}=5.7$; one participant did not provide age information). All participants had normal or corrected-tonormal vision and did not report any history of mental illness. All participants provided informed consent and course credits or a monetary reward was provided as compensation. Procedures were approved by the local ethics committee, and in accordance with the Declaration of Helsinki ("WMA Declaration of Helsinki - Ethical Principles for Medical Research Involving Human Subjects," 2013).

\subsection{Materials and design}

\subsubsection{Psychopathy questionnaire}

Participants started the experiment by completing the Psychopathic Personality Inventory (PPI), a self-report questionnaire of 187 items that assesses psychopathic traits (Lilienfeld \& Andrews, 1996). The overall PPI score can be split into two specific factors associated with psychopathy: PPI-I has been labeled 'Fearless Dominance' and represents social potency, fearlessness and stress immunity, whereas PPIII is called 'Impulsive Antisociality' and refers to impulsivity, egocentricity and aggressiveness among the most dominant traits. These 
factors have been found to be highly valid, demonstrating relations with psychopathic traits (Benning, Patrick, Blonigen, Hicks, \& Iacono, 2005; Benning, Patrick, Hicks, Blonigen, \& Krueger, 2003).

\subsubsection{Emotional capture task}

Following the questionnaire, participants conducted the Emotional Capture Task. Stimulus presentation and response collection were managed by a PC with a 22" monitor, using Matlab 2014b with the Psychophysics Toolbox extension (Brainard, 1997; Pelli, 1997). Stimuli were presented at a viewing distance of $75 \mathrm{~cm}$, maintained with a chinrest. The stimuli were the same as those used by Hodsoll et al. (2014), consisting of a subset of faces selected from the NimStim Face Stimulus Set (Tottenham et al., 2009). These faces consisted of twelve different identities: Six male and six female faces, each identity having a happy, neutral and fearful face. The face images were used in two separate tasks: The orientation task and the emotion task.

The orientation task was similar to the task used by Hodsoll et al. (2014). Participants were shown three images of different faces, presented around a central fixation dot (visual angle $=0.3^{\circ}$ ). All images subtended a visual angle of $4.19^{\circ} \times 2.82^{\circ}(\mathrm{h} \times \mathrm{w})$, and were presented $2.67^{\circ}$ away from fixation (inner edge). All faces could be presented perfectly upright $\left(0^{\circ}\right.$ angle) or presented at a $15^{\circ}$ angle to the left or right. Two faces were always presented above fixation and one face was presented below fixation (see Fig. 1). One of the presented faces would always have a male identity which served as the target stimulus. Of the three presented faces, two faces would display neutral facial expressions, whereas the remaining face could display one of the three emotional expressions: Happy, fearful or neutral. The experiment was designed such that two-thirds of the trials contained an emotional expression at the male target face (happy or fearful). Of the remaining trials, when the target face displayed a neutral expression, two thirds of those trials contained a female distractor face displaying a happy or fearful facial expression. Therefore, one ninth of the trials contained only faces with neutral facial expressions. All experimental factors were counterbalanced and randomized within blocks. In the orientation task, participants were instructed to indicate as fast as possible whether the male face was tilted to the left or right or presented upright, by pressing one of three keys on a standard keyboard ('v', 'b' and ' $n$ ' for left tilted, upright and right tilted). The emotion task was similar to the orientation task: Participants were instructed to search for the male target face and respond as fast as possible to whether this face displayed a neutral, happy or fearful emotional expression by pressing the ' $v$ ', ' $b$ ' and ' $n$ ' keys, respectively.

Participants were tested in a darkened room, and performed both tasks (orientation and emotion) in one session. The emotion and orientation task were presented in a blocked fashion, their order counterbalanced. For each trial, a central fixation dot appeared for $0.5 \mathrm{~s}$, which was then followed by the search display which remained on the screen until a response was made. For either task, participants completed a practice block of 24 trials, then proceeded to complete four experimental blocks of 81 trials each (324 experimental trials in total).

\subsubsection{Data analysis}

Separate analyses of variance (ANOVAs) on the reaction time data were conducted on trials in which the emotion was presented on the target face versus trials on which the emotion was presented on the distractor face. As the RMH clearly states that emotion processing deficits arise when important environmental stimuli are irrelevant to goal-directed behavior, a difference between target and distractor emotions was expected.

One-ninth of all trials consisted of only neutral faces. These trials were not entered into the ANOVA, due to being physically identical when the neutral emotion was presented at the target or a distractor face. Therefore, initial analyses were only conducted on trials containing either a happy or a fearful emotion on one of the faces. Subsequently, post-hoc comparisons were conducted against the appropriate neutral condition for baseline comparisons (i.e. the average reaction time for all neutral trials independently for each task).

To summarize, we conducted a series of mixed model ANOVAs on the reaction time data, with task (orientation, emotion) and emotion (happy, fearful) as within-subjects factors and the level of psychopathy as a between-subjects factor. Because psychopathy is a dimensional construct (Edens, Marcus, Lilienfeld, \& Poythress, 2006), two groups were created based on a median split of the factorial PPI scores. While a loss of power is unavoidable, this ensures that the different groups have significantly different psychopathy scores. In addition, it is the only option available to split out an interaction between task, emotion and psychopathy. The results below focus only on interactions between the experimental factors and psychopathy scores. For overall task performance, independent of psychopathy, see Supplementary Fig. S1.

\section{Results}

Data from one participant was removed due to poor behavioral performance on the orientation task. For the reaction times analyses, all trials with incorrect responses were removed from the data set (6.56\%). Subsequently, the data was trimmed towards a normal distribution by removing all trials in which the participant responded slower than $3000 \mathrm{~ms}$ or faster than $200 \mathrm{~ms}(0.81 \%)$. Additionally, all trials were removed with reaction times $2.5 \mathrm{SD}$ above the average of the trimmed data, separately for each participant and task (2.71\%). Table 1 describes the properties of the psychopathy questionnaire.
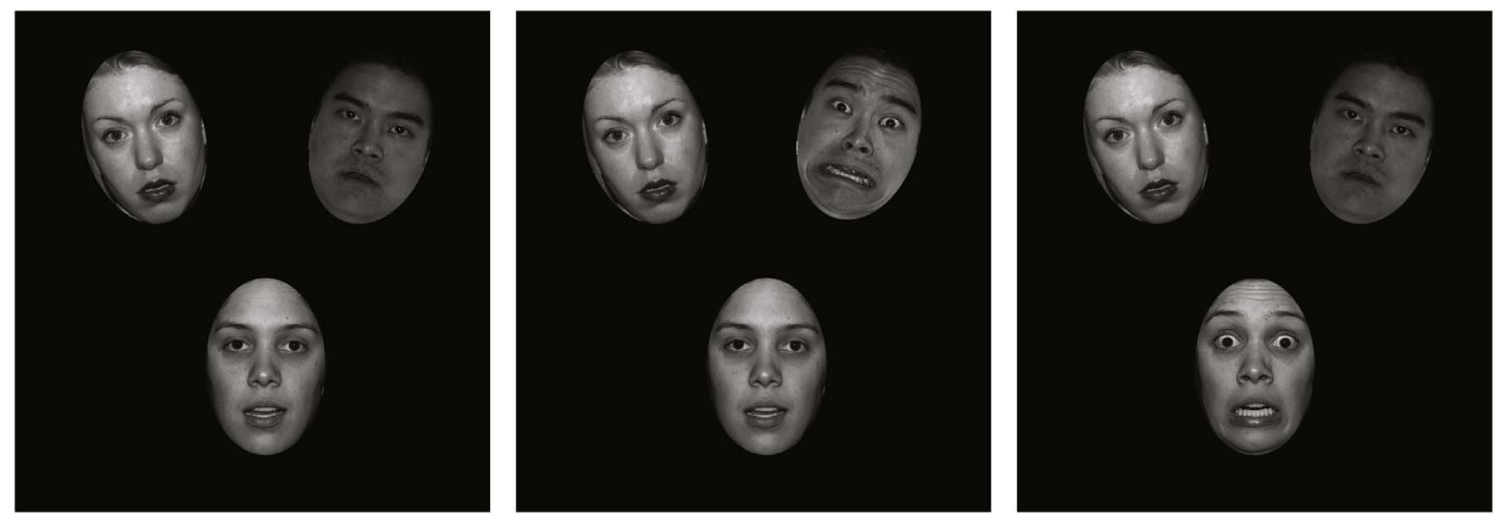

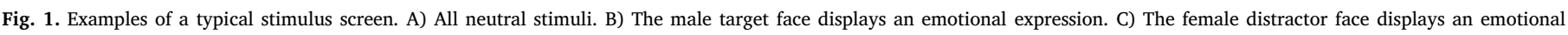
expression. 
Table 1

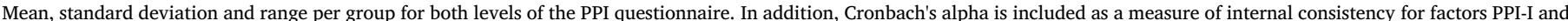
PPI-II.

\begin{tabular}{|c|c|c|c|c|c|c|c|}
\hline & \multirow[t]{2}{*}{ Cronbach's alpha } & \multicolumn{3}{|c|}{ Low psychopathy group } & \multicolumn{3}{|c|}{ Elevated psychopathy group } \\
\hline & & Mean & SD & Range & Mean & SD & Range \\
\hline PPI-I & 0.899 & 119.43 & 13.35 & $81-135$ & 151.12 & 11.48 & $136-191$ \\
\hline PPI-II & 0.898 & 157.10 & 12.81 & $119-173$ & 198.39 & 16.70 & $174-235$ \\
\hline
\end{tabular}

Table 2

Reaction times and accuracy for each condition, separately for the low scoring and the elevated group for the two different PPI factors (PPI-I and PPI-II).

\begin{tabular}{|c|c|c|c|c|c|c|c|c|}
\hline & \multicolumn{4}{|c|}{ Low scoring group } & \multicolumn{4}{|c|}{ Elevated group } \\
\hline & \multicolumn{2}{|l|}{ Happy } & \multicolumn{2}{|l|}{ Fearful } & \multicolumn{2}{|l|}{ Happy } & \multicolumn{2}{|l|}{ Fearful } \\
\hline & RT (ms) & Acc $(\%)$ & RT (ms) & Acc (\%) & $\mathrm{RT}$ (ms) & Acc (\%) & RT (ms) & Acc $(\%)$ \\
\hline \multicolumn{9}{|l|}{ PPI-I } \\
\hline \multicolumn{9}{|l|}{ Emotion task } \\
\hline Target & $958(24)$ & $92.6(0.9)$ & $1036(30)$ & $92.7(1.1)$ & 967 (19) & $95.0(0.5)$ & $1076(24)$ & $92.4(0.8)$ \\
\hline Distractor & 1089 (29) & $91.0(1.4)$ & $1110(30)$ & $89.1(1.5)$ & $1118(31)$ & $92.7(1.0)$ & $1143(27)$ & $92.3(1.0)$ \\
\hline \multicolumn{9}{|c|}{ Orientation task } \\
\hline Target & 751 (19) & $94.1(0.8)$ & 760 (19) & $93.9(0.8)$ & $742(12)$ & $95.2(0.6)$ & 753 (13) & $94.7(0.7)$ \\
\hline Distractor & $785(22)$ & $94.1(0.8)$ & $803(22)$ & $92.4(1.0)$ & $784(15)$ & $94.5(0.8)$ & 799 (15) & $94.2(1.0)$ \\
\hline \multicolumn{9}{|l|}{ PPI-II } \\
\hline \multicolumn{9}{|l|}{ Emotion task } \\
\hline Target & $961(24)$ & $94.5(0.6)$ & 1047 (28) & $93.3(0.8)$ & $964(20)$ & $93.2(0.8)$ & $1066(26)$ & $91.8(1.1)$ \\
\hline Distractor & $1112(34)$ & $91.8(1.0)$ & 1113 (29) & $90.8(1.1)$ & $1096(26)$ & $91.8(1.4)$ & $1140(28)$ & $90.6(1.5)$ \\
\hline \multicolumn{9}{|c|}{ Orientation task } \\
\hline Target & 741 (14) & $95.2(0.5)$ & $753(15)$ & $95.3(0.6)$ & $751(18)$ & $94.1(0.8)$ & 759 (17) & $93.5(0.8)$ \\
\hline Distractor & 779 (18) & $94.5(0.6)$ & $801(16)$ & $94.3(0.8)$ & 791 (19) & $94.1(0.8)$ & $801(21)$ & $92.4(1.1)$ \\
\hline
\end{tabular}

\subsection{Psychopathy analyses}

\subsubsection{Emotion at target}

The mixed model ANOVA on the reaction times from trials in which the male target face displayed an emotional expression did not show any interactions between psychopathy and the experimental factors (smallest $p$ 's: PPI-I $=0.135$; PPI-II $=0.296$; See Table 2 for all reaction times).

\subsubsection{Emotion at distractor}

Using PPI-I as a between-subjects factor did not yield a task $\mathrm{x}$ emotion $\mathrm{x}$ PPI-I interaction $(F<1)$, whereas this three-way interaction was highly significant when using PPI-II as a between-subjects factor $(F$ $\left.(1,97)=7.895, p=0.006, \eta_{p}{ }^{2}=0.075\right)$. Post-hoc ANOVAs with emotion as a within-subjects factor and PPI-II as a between-subjects factor, conducted separately per task, failed to produce a significant emotion $\mathrm{x}$ PPI-II interaction in the orientation task $(F(1,97)=1.153$, $p=0.286, \eta_{p}{ }^{2}=0.012$ ), but did result in a significant interaction between emotion $\mathrm{x}$ PPI-II in the emotion task $(F(1,97)=6.792$, $\left.p=0.011, \eta_{p}{ }^{2}=0.065\right)$. Post-hoc $t$-tests showed that the difference in reaction times between trials with happy and fearful distractor facial expressions was small and non-significant for the low-scoring psychopathy group $(\Delta 1 \mathrm{~ms}, t(47)=0.086, p=0.932)$, whereas this difference was large and significant for the elevated psychopathy group ( $\Delta 44 \mathrm{~ms}, t$ $(50)=3.811, p<0.001)$. Participants in the elevated psychopathy group responded slowest to the male target face when one of the female distractor faces displayed a fearful emotional expression (Fig. 2). As the only statistically significant interactions between psychopathy and the experimental factors arose from using PPI-II as a between-subjects factor, we will be using this factor for the remaining analyses.

\subsubsection{Post-hoc comparisons with neutral trials}

To disentangle the differences between the low and elevated psychopathy groups with regards to emotional attentional capture by distracting emotional faces, we compared the reaction times from trials with happy or fearful distractor faces to trials consisting of only neutral faces. As previous analyses showed that the level of psychopathy only interacted with emotion in the emotion task, we conducted these posthoc analyses for this task alone and solely for trials in which the distractor face displayed an emotional expression.

Fig. 2 shows the average reaction times for the happy, fearful and neutral distractor trials during the emotion task. Post-hoc tests showed that reaction times to neutral trials in the emotion task were significantly faster than reaction times to distractors displaying a happy $(\Delta 40 \mathrm{~ms} ; p=0.004)$ or a fearful $(\Delta 41 \mathrm{~ms} ; p=0.001)$ expression in the low psychopathy group. Contrary to these findings, post-hoc analyses on the reaction times in the emotion task indicated that the elevated group showed faster response times only to trials with distractor faces displaying fearful emotional expressions ( $\Delta 66 \mathrm{~ms} ; p<0.001)$, but this test did not reach significance for happy emotional expressions ( $\Delta 22 \mathrm{~ms} ; p=0.094$ ). Importantly, the amount of emotional attentional capture for the elevated psychopathy group appeared to be much stronger for fearful facial expressions as compared to happy facial expressions, as indicated by a significant difference between the two emotions $(p<0.001)$. For the low scoring psychopathy group, no such difference was observed $(p=0.932)$.

No effects of emotional attentional capture were observed in the accuracy data. Neither the low scoring psychopathy group, nor the elevated psychopathy group showed a difference in accuracy between neutral, happy and fearful trials in the emotion task when presented on a distractor face (low scoring group: smallest $p=0.224$; elevated group: smallest $p=0.194$ ).

\section{Discussion}

To better understand the interplay between emotional and cognitive factors in psychopathy, we compared two theories on the relation between psychopathy and attentional allocation to emotional facial 


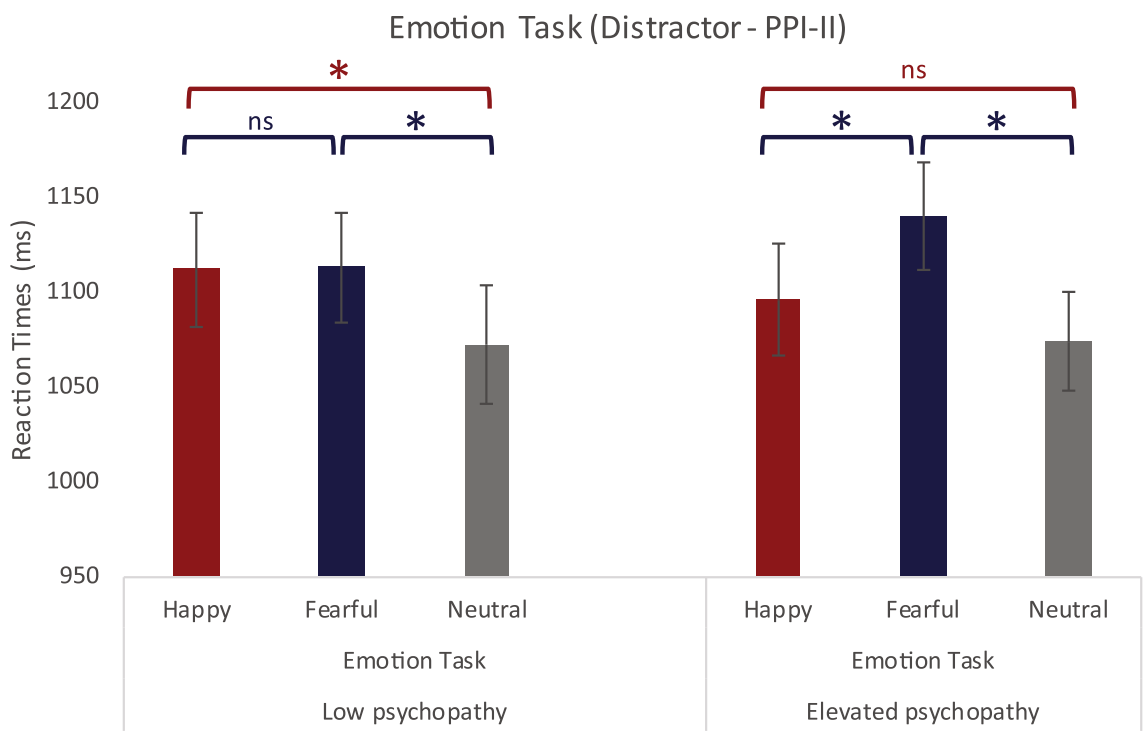

Fig. 2. The two levels of psychopathy display a different pattern of emotional attentional capture for happy and fearful emotional expressions when emotion is task relevant and displayed on a distractor face. Error bars reflect the standard error of the mean. expressions. Here, participants conducted two tasks in which they either indicated the orientation or the emotion of a target face.

The current study focused on 1) investigating to what extent topdown attentional set influenced emotional attentional capture in elevated compared to low scoring participants and 2) investigating how different emotional expressions may lead to different patterns of emotional attentional capture between elevated and low scoring psychopathic individuals. The first main finding is that no differences between the two groups were observed when the target face displayed an emotional expression. The second main finding is that top-down set influenced the degree to which an emotional facial expression attracted attention between the low versus elevated psychopathy group. That is, when emotion was task-relevant, the elevated psychopathy group showed no emotional attentional capture by happy distractor faces whereas this was the case in the low psychopathy group. When emotion was task-relevant, both groups showed similar responses to a fearful distractor. So, where the IES would predict abnormalities in the detection of fearful faces (be it as a target or distractor), no such pattern was observed here. Therefore, these findings suggest that top-down set has an influence on attentional allocation towards important emotional stimuli, which aligns with the RMH. However, some deviations were observed here: The RMH postulates that all stimuli not fitting a psychopathic individual's active top-down set will not be attended as they are irrelevant to their ongoing goals. Yet, when emotion was part of the top-down set individuals with elevated psychopathic traits were captured by fearful, but not happy distractor faces. This difference between groups was not observed when emotion was task-irrelevant. As such, these findings further suggest that cognitive factors (i.e., attention, topdown set) may not fully explain all emotion processing abnormalities in psychopathy (see Supplementary section S2 for an alternative explanation). The concept of top-down attention as an underlying factor in psychopathy may therefore benefit from further refinement.

The observation that different emotions lead to different patterns of attentional capture, provides a tentative indication as to how the RMH can be refined. Deficits in attentional deployment to emotional faces, may potentially interact with the nature of the task being conducted even when these tasks rely on a similar top-down set. The current study investigated emotional attentional capture in a rapid visual search task, leading to differential responses for happy and fearful faces. In previous work, Baskin-Sommers and Newman (2014) used an attentional task in which the behavioral response was more intimately linked with the categorization of emotional expressions. In their study, no effect of the type of emotion was observed on attentional guidance. Therefore, it may be the case that both top-down attentional set as well as task-type determine to what extent psychopathic individuals are captured by emotional expressions.

A crucial observation is that all observed effects of psychopathy rely on PPI-II scores. PPI-II reflects behavioral traits that are labeled as 'Impulsive Antisociality', referring to impulsivity, egocentricity and aggressiveness. No effects of psychopathy were observed for PPI-I scores. The observation that cognitive factors play a major role in psychopathy as defined by PPI-II is in line with a recent meta-analysis that showed that structural and functional deficits in dorsolateral prefrontal cortex (DLPFC) of antisocial, psychopathic individuals correlated with poorer attentional control (Yang \& Raine, 2009). In line with this finding, Sadeh and Verona (2008) showed reduced processing of irrelevant distractors for psychopathic individuals with primary psychopathic traits (e.g. callousness \& social dominance). This is in line with the current observation that participants with elevated psychopathic traits were less distracted by happy emotional distractor faces. However, in the current study mainly PPI-II interacted with attention to influence the processing of emotional distractor faces, preventing the formulation of a clear conclusion as to which specific psychopathic traits interact with attention and emotion.

Despite a carefully constructed experimental design, some limitations should be addressed. First, the factor structure (i.e. PPI-I and PPIII) of a questionnaire may vary when administered in different populations. However, given the current sample size it is not possible to conduct confirmatory factor analyses which leaves open the possibility that the factor structure in this sample is not similar to that reported in prior publications. Nonetheless, Cronbach's alpha was observed to be high for both factors, providing evidence that the test items making up the factors consistently measured the questionnaire's latent underlying variables. A second limitation comes from using a small sample. Although the sample size is sufficient for simple psychophysical measurements, the inclusion of a between subjects-factor related to highlyvariant personality properties resulted in a sample that may not be large enough to expose all effects present in the data. However, the current study does provide an excellent starting point for future studies in larger, well-powered samples.

\section{Declaration of conflicting interests}

The author(s) declared no potential conflicts of interest with respect to the research, authorship, and/or publication of this article. 


\section{Acknowledgements}

The authors would like to thank Stefan van der Stigchel for his helpful comments and suggestions.

\section{Funding}

The author(s) disclosed receipt of the following financial support for the research, authorship, and/or publication of this article: Preparation of this manuscript was supported by an ERC advanced grant [ERC2012-AdG - 323413 Jan Theeuwes] and a TUBITAK-BIDEB visiting scientist grant [2221 - J. Munneke].

\section{Appendix A. Supplementary data}

Supplementary data to this article can be found online at https:// doi.org/10.1016/j.paid.2017.10.019.

\section{References}

Baskin-Sommers, A. R., Curtin, J. J., \& Newman, J. P. (2011). Specifying the attentional selection that moderates the fearlessness of psychopathic offenders. Psychological Science, 22. http://dx.doi.org/10.1177/0956797610396227.

Baskin-Sommers, A. R., \& Newman, J. P. (2014, October). Psychopathic and externalizing offenders display dissociable dysfunctions when responding to facial affect. Personality Disorders, 5(4), 369-379. http://dx.doi.org/10.1037/per0000077.

Benning, S. D., Patrick, C. J., Blonigen, D. M., Hicks, B. M., \& Iacono, W. G. (2005) Estimating facets of psychopathy from normal personality traits: A step toward community epidemiological investigations. Assessment, 12, 3-18. http://dx.doi.org/ 10.1177/1073191104271223.

Benning, S. D., Patrick, C. J., Hicks, B. M., Blonigen, D. M., \& Krueger, R. F. (2003). Factor structure of the psychopathic personality inventory: Validity and implications for clinical assessment. Psychological Assessment, 15, 340-350. http://dx.doi.org/10. 1037/1040-3590.15.3.340.

Blair, R. J. R. (2005). Applying a cognitive neuroscience perspective to the disorder of psychopathy. Development and Psychopathology, 17, 865-891. http://dx.doi.org/10. 1017/S0954579405050418.

Blair, R. J. R., \& Mitchell, D. G. V. (2009). Psychopathy, attention and emotion. Psychological Medicine, 39, 543-555. http://dx.doi.org/10.1017/ S0033291708003991.

Blair, R. J. R., Mitchell, D. G. V., Peschardt, K. S., Colledge, E., Leonard, R. A., Shine, J. H., ... Perrett, D. I. (2004). Reduced sensitivity to others' fearful expressions in psychopathic individuals. Personality and Individual Differences, 37, 1111-1122. http://dx. doi.org/10.1016/j.paid.2003.10.008

Brainard, D. H. (1997). The psychophysics toolbox. Spatial Vision, 10, 433-436. http://dx. doi.org/10.1163/156856897X00357.

Byrne, A., \& Eysenck, M. W. (1995). Trait anxiety, anxious mood, and threat detection Cognition and Emotion, 9, 549-562. http://dx.doi.org/10.1080/02699939508408982.

Dawel, A., O'Kearney, R., McKone, E., \& Palermo, R. (2012). Not just fear and sadness: Meta-analytic evidence of pervasive emotion recognition deficits for facial and vocal expressions in psychopathy. Neuroscience \& Biobehavioral Reviews, 36, 2288-2304. http://dx.doi.org/10.1016/j.neubiorev.2012.08.006.

Edens, J. F., Marcus, D. K., Lilienfeld, S. O., \& Poythress, N. G. (2006). Psychopathic, not psychopath: Taxometric evidence for the dimensional structure of psychopathy. Journal of Abnormal Psychology, 115, 131-144. http://dx.doi.org/10.1037/0021 843X.115.1.131.

Fairchild, G., Stobbe, Y., van Goozen, S. H. M., Calder, A. J., \& Goodyer, I. M. (2010). Facial expression recognition, fear conditioning, and startle modulation in female subjects with conduct disorder. Biological Psychiatry, 68, 272-279. http://dx.doi.org/ 10.1016/j.biopsych.2010.02.019.

Fairchild, G., Van Goozen, S. H. M., Calder, A. J., Stollery, S. J., \& Goodyer, I. M. (2009) Deficits in facial expression recognition in male adolescents with early-onset or adolescence-onset conduct disorder. Journal of Child Psychology and Psychiatry, 50, 627-636. http://dx.doi.org/10.1111/j.1469-7610.2008.02020.x.

Glass, S. J., \& Newman, J. P. (2006). Recognition of facial affect in psychopathic offenders. Journal of Abnormal Psychology, 115, 815-820. http://dx.doi.org/10.1037/ 0021-843X.115.4.815.

Gorenstein, E. E., \& Newman, J. P. (1980). Disinhibitory psychopathology: A new perspective and a model for research. Psychological Review, 87, 301-315. http://dx.doi. org/10.1037/0033-295X.87.3.301.

Hare, R. D. (1991). The Hare psychopathy checklist-revised: Manual. Toronto, ON: MultiHealth Systems, Incorporated.

Hodsoll, S., Lavie, N., \& Viding, E. (2014, August 26). Emotional attentional capture in children with conduct problems: The role of callous-unemotional traits. Frontiers in Human Neuroscience, 8. http://dx.doi.org/10.3389/fnhum.2014.00570.

Hoppenbrouwers, S., van der Stigchel, S., Slotboom, J., Dalmeijer, E., \& Theeuwes, J. (2015). Disentangling attentional deficits in psychopathy using visual search: Failures in the use of contextual information. Personality and Individual Differences, 86, 132-138. http://dx.doi.org/10.1016/j.paid.2015.06.009.

Hoppenbrouwers, S. S., Bulten, B. H., \& Brazil, I. A. (2016). Parsing fear: A reassessment of the evidence for fear deficits in psychopathy. Psychological Bulletin, 142(6), 573-600. http://dx.doi.org/10.1037/bul0000040.

Hoppenbrouwers, S. S., Van der Stigchel, S., Sergiou, C. S., \& Theeuwes, J. (2016). Topdown attention and selection history in psychopathy: Evidence from a community sample. Journal of Abnormal Psychology, 125, 435-441. http://dx.doi.org/10.1037/ abn0000133.

Lilienfeld, S. O., \& Andrews, B. P. (1996). Development and preliminary validation of a self-report measure of psychopathic personality traits in noncriminal population. Journal of Personality Assessment, 66, 488-524. http://dx.doi.org/10.1207/ s15327752jpa6603 3 .

Marsh, A. A., \& Blair, R. J. R. (2008). Deficits in facial affect recognition among antisocial populations: A meta-analysis. Neuroscience \& Biobehavioral Reviews, 32, 454-465. http://dx.doi.org/10.1016/j.neubiorev.2007.08.003.

Newman, J. P., \& Baskin-Sommers, A. R. (2012). Early selective attention abnormalities in psychopathy. Cognitive neuroscience of attention (pp. 421-440). .

Newman, J. P., Curtin, J. J., Bertsch, J. D., \& Baskin-Sommers, A. R. (2010). Attention moderates the fearlessness of psychopathic offenders. Biological Psychiatry, 67, 66-70. http://dx.doi.org/10.1016/j.biopsych.2009.07.035.

Newman, J. P., \& Wallace, J. F. (1993). Diverse pathways to deficient self-regulation: Implications for disinhibitory psychopathology in children. Clinical Psychology Review, 13, 699-720. http://dx.doi.org/10.1016/S0272-7358(05)80002-9.

Patterson, M. C., \& Newman, J. P. (1993). Reflectivity and learning from aversive events: Toward a psychological mechanism for the syndromes of disinhibition. Psychological Review, 100, 716-736. http://dx.doi.org/10.1037/0033-295X.100.4.716.

Pelli, D. G. (1997). The VideoToolbox software for visual psychophysics: Transforming numbers into movies. Spatial Vision, 10, 437-442. http://dx.doi.org/10.1163/ 156856897 X00366.

Sadeh, N., \& Verona, E. (2008). Psychopathic personality traits associated with abnormal selective attention and impaired cognitive control. Neuropsychology, 22, 669-680. http://dx.doi.org/10.1037/a0012692.

Tottenham, N., Tanaka, J. W., Leon, A. C., McCarry, T., Nurse, M., Hare, T. A., ... Nelson, C. (2009). The NimStim set of facial expressions: Judgments from untrained research participants. Psychiatry Research, 168, 242-249. http://dx.doi.org/10.1016/j. psychres.2008.05.006.

Viding, E., \& McCrory, E. J. (2012). Genetic and neurocognitive contributions to the development of psychopathy. Development and Psychopathology, 24, 969-983. http:// dx.doi.org/10.1017/S095457941200048X.

Yang, Y., \& Raine, A. (2009). Prefrontal structural and functional brain imaging findings in antisocial, violent, and psychopathic individuals: A meta-analysis. Psychiatry Research: Neuroimaging, 174, 81-88. http://dx.doi.org/10.1016/j.pscychresns.2009. 03.012.

Yiend, J. (2010). The effects of emotion on attention: A review of attentional processing of emotional information. Cognition and Emotion, 24, 3-47. http://dx.doi.org/10. 1080/02699930903205698. 